\title{
Incidence of Febrile Neutropenia in Pediatric Solid Tumor and Lymphoma Patients treated with Chemotherapy
}

Ah Young Jeon ${ }^{1}$, Nayoung Han², Myeong Gyu Kim³, Yoonsook Cho', Eun Jeong Cho', Min Jung Kim¹, A Jeong Kim¹, Hyoung Jin Kang4, Jung Mi Oh², Hee Young Shin ${ }^{4}$

1Department of Pharmacy, Seoul National University Hospital, Seoul, Republic of Korea

${ }^{2}$ College of Pharmacy and Research Institute of Pharmaceutical Sciences, Seoul National University, Seoul, Republic of Korea

${ }^{3}$ Graduate School of Clinical Pharmacy, CHA University, Pocheon, Republic of Korea

${ }^{4}$ Department of Pediatrics, Cancer Research Institute, Seoul National University College of Medicine, Seoul, Republic of Korea

\section{Introduction}

- Febrile neutropenia (FN) is a potentially life-threatening complication of cancer treatments.

- Many guidelines (NCCN, ASCO, EORTC) recommend prophylactic use of granulocyte colony-stimulating factor (GCSF) if the risk of $\mathrm{FN}$ is greater than $20 \%$ and are presenting lists of chemotherapy regimens with high risk of $\mathrm{FN}^{1,2,3}$

- However, information regarding the incidence of FN among different chemotherapy regimens for pediatric patients is scarce.

\section{Objectives}

- We aimed to evaluate incidence of FN in pediatric solid tumor and lymphoma patients treated with chemotherapy and assess risk factors in each tumor type and regimen.

\section{Methods}

Study design

- Retrospective observational study

Patients

- Pediatric solid tumor and lymphoma patients newly administered chemotherapy from 2011 to 2015 in Seoul National University Children's Hospital

Outcomes

- Primary outcome

- Time-dependent cumulative incidence

(Cl) of FN with different tumor types and chemotherapy regimens

- Secondary outcome - Risk factors for FN

Statistics

- R function to obtain $\mathrm{Cl}$ according to chemotherapy regimens

- Cox regression to analyze FN incidence regarding different combinations of chemotherapeutic agents

- Univariate logistic regression for risk factor analysis

\section{Results}

- Of the 409 patients, the most common tumor type was central nervous system (CNS) tumor (27\%).

- Non-hodgkin's lymphoma (NHL) (16\%), retinoblastoma (16\%), bone cancer $(10 \%)$, neuroblastoma $(9 \%)$, rhabdomyosarcoma (6\%), hepato-biliary cancer (4\%), wilms tumor (4\%), germ cell tumor (GCT) (4\%), hodgkin's lymphoma $(\mathrm{HL})(3 \%)$, soft tissue sarcoma $(1 \%)$

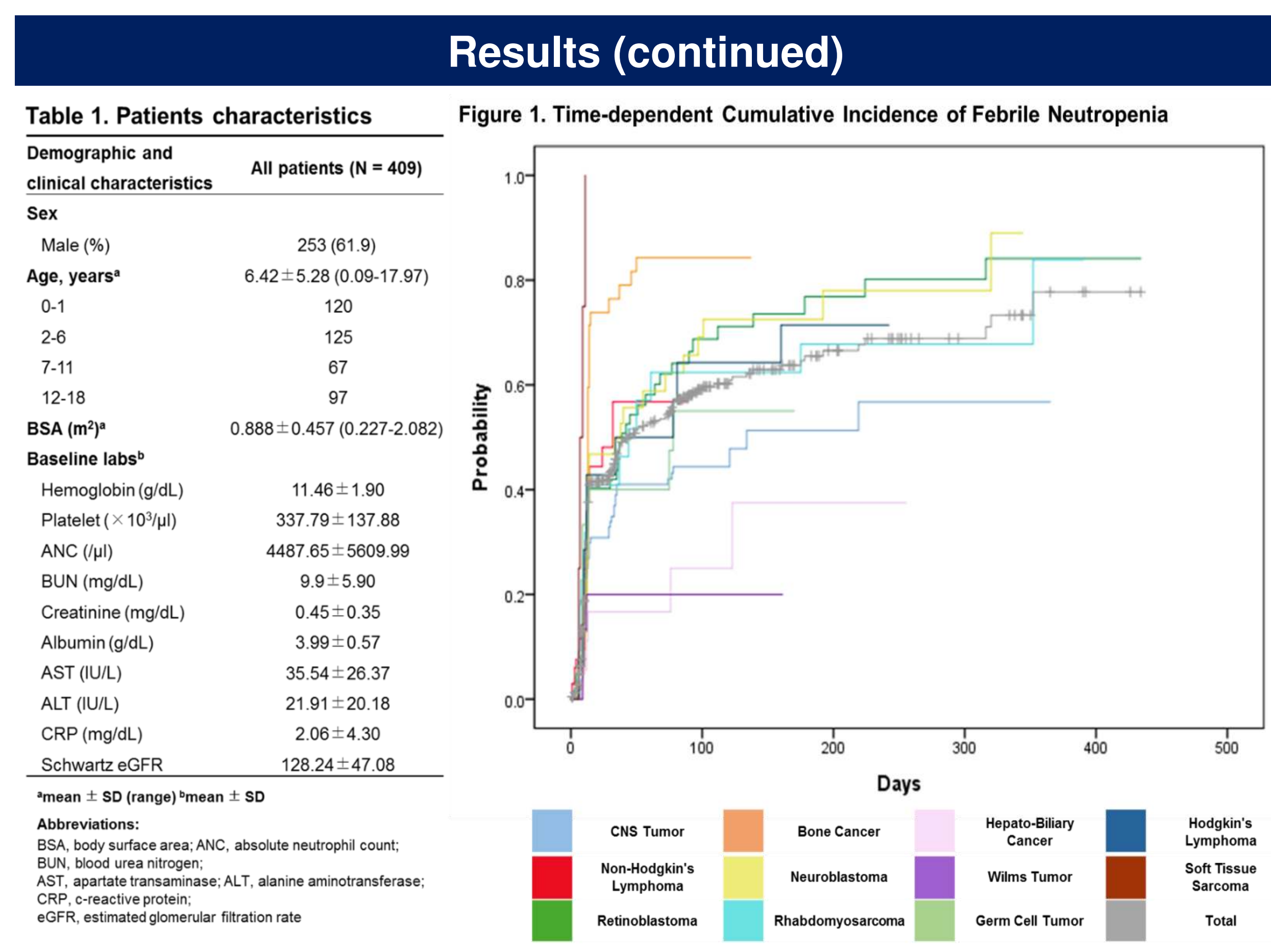

- Incidence of FN were $45.5 \%$ in KSPNO regimen for low risk CNS GCT $(\mathrm{Cl}=0.520), 4.5 \%$ in 9952A for low grade glioma ( $\mathrm{Cl}=0.048), 6.3 \%$ in 5961 modified-COP for $\mathrm{NHL}(\mathrm{Cl}=0.063)$, 80.0\% in CarDEC for retinoblastoma ( $\mathrm{Cl}=0.906), 87.0 \%$ in CCG 7921A for bone cancer $(\mathrm{Cl}=0.870)$, and $78.8 \%$ in $\mathrm{CCG} 321 \mathrm{P} 2$ for neuroblastoma $(\mathrm{Cl}=1.000)$.

Table 2. Incidence of Febrile Neutropenia treated with Chemotherapy regimen (consisting of more than 10 patients)

\begin{tabular}{|c|c|c|c|c|c|}
\hline Tumor type & Chemotherapy regimen & $\begin{array}{l}\text { Combinations of } \\
\text { chemotherapeutic agents }\end{array}$ & $\begin{array}{c}\text { All } \\
\text { patients }\end{array}$ & $\mathrm{FN}(\%)$ & $\begin{array}{c}\text { Cumulative } \\
\text { incidence (Cl) }\end{array}$ \\
\hline \multirow{5}{*}{ CNS Tumor } & 9952A (Induction) & CARB-VCR & 22 & $1(4.5)$ & 0.048 \\
\hline & KSPNO For Low risk CNS GCT & CARB-CPM-VP & 22 & $10(45.5)$ & 0.520 \\
\hline & CCRT wtih TMZ & TMZ & 17 & $1(5.9)$ & 0.125 \\
\hline & KSPNO-S-1102A & CDDP-CPM-VP-VCR & 14 & $14(100.0)$ & 1.000 \\
\hline & KSPNO For High risk CNS GCT & BLM-CARB-CPM-VP & 13 & $9(69.2)$ & 0.892 \\
\hline \multirow{3}{*}{ Non-Hodgkin's Lymphoma } & 5961 modified-COP & CPM-PD-VCR & 32 & $2(6.3)$ & 0.063 \\
\hline & 106B (Induction) & CPM-DNR-LASP-PD-VCR & 16 & $15(93.8)$ & 0.938 \\
\hline & (D)-COMP (Induction) & CPM-MTX-PD-VCR & 12 & $1(8.3)$ & 0.083 \\
\hline Retinoblastoma & CarDEC & CARB-CPM-ADR-VP-VCR & 45 & $36(80.0)$ & 0.906 \\
\hline \multirow{2}{*}{ Bone Cancer } & CCG 7921A (Induction) & CDDP-ADR-MTX & 23 & $20(87.0)$ & 0.870 \\
\hline & VDC & CPM-ADR-VCR & 12 & $11(91.7)$ & 1.000 \\
\hline Neuroblastoma & CCG 321P2 & CDDP-CPM-ADR-VP & 33 & $26(78.8)$ & 1.000 \\
\hline Rhabdomyosarcoma & POG-ICE & CARB-VP-IFO & 18 & $12(66.7)$ & 0.832 \\
\hline Hepato-Biliary Cancer & $8881 \mathrm{~A}$ & CDDP-VCR-5FU & 15 & $2(13.3)$ & 0.222 \\
\hline Germ Cell Tumor & CCG 8882 (Induction) & BLM-CDDP-VP & 12 & $7(58.3)$ & 0.583 \\
\hline
\end{tabular}

DNR, dauorubicin; LASP, I-asparaginase; MTX, methotrexate; ADR, doxorubicin; IFO, ifosfamide; $5 F U, 5$-fluorouracil

- FN occurred mainly in the first cycle of chemotherapy in most regimens.

- With regard to combinations of chemotherapeutic agents, incidence of FN after treatment with CPM/DNR/LASP/PD/VCR was significantly high compared to CPM/PD/VCR ( $H R=6.998$, $p=0.012)$ in NHL.

- Low hemoglobin $(\leq 12 \mathrm{~g} / \mathrm{dL})$ was the risk factor of FN in CNS tumor treated with KSPNO regimen for low risk CNS GCT $(O R=16.5, p=0.022)$.

\section{Conclusions}

- Incidence of FN in pediatric solid tumor and lymphoma patients is largely dependent on tumor type and chemotherapy regimens.

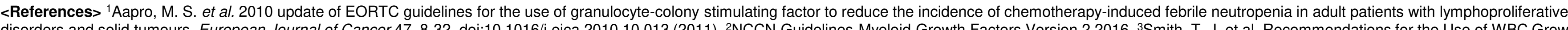

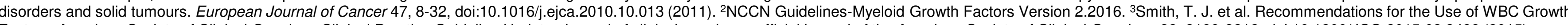

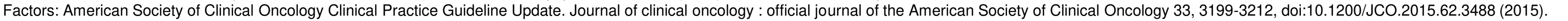

